Damage-resistant single-pulse optics for x-ray free electron lasers

Stefan Hau-Riege, Richard London, Michael Bogan, Henry Chapman, Magnus Bergh

May 8, 2007

Optics and Optoelectronics

Prague, Czech Republic

April 16, 2007 through April 19, 2007 
This document was prepared as an account of work sponsored by an agency of the United States Government. Neither the United States Government nor the University of California nor any of their employees, makes any warranty, express or implied, or assumes any legal liability or responsibility for the accuracy, completeness, or usefulness of any information, apparatus, product, or process disclosed, or represents that its use would not infringe privately owned rights. Reference herein to any specific commercial product, process, or service by trade name, trademark, manufacturer, or otherwise, does not necessarily constitute or imply its endorsement, recommendation, or favoring by the United States Government or the University of California. The views and opinions of authors expressed herein do not necessarily state or reflect those of the United States Government or the University of California, and shall not be used for advertising or product endorsement purposes. 


\title{
Damage-resistant single-pulse optics for $x$-ray free electron lasers
}

\author{
Stefan P. Hau-Riege ${ }^{a}$, Richard A. London ${ }^{\mathrm{a}}$, Michael Bogan ${ }^{\mathrm{a}}$, Henry N. Chapman ${ }^{\mathrm{a}}$, \\ and Magnus Bergh ${ }^{\mathrm{b}}$ \\ ${ }^{a}$ Lawrence Livermore National Laboratory, P.O. Box 808, Livermore, CA 94551; \\ ${ }^{b}$ Biomedical Center, Uppsala University, Box 576, SE 75123, Uppsala, Sweden
}

\begin{abstract}
Short-pulse ultraviolet and x-ray free electron lasers of unprecedented peak brightness are in the process of revolutionizing physics, chemistry, and biology. Optical components for these new light sources have to be able to withstand exposure to the extremely high-fluence photon pulses. Whereas most optics have been designed to stay intact for many pulses, it has also been suggested that single-pulse optics that function during the pulse but disintegrate on a longer timescale, may be useful at higher fluences than multiple-pulse optics. In this paper we will review damageresistant single-pulse optics that recently have been demonstrated at the FLASH soft-X-ray laser facility at DESY, including mirrors, apertures, and nanolenses. It was found that these objects stay intact for the duration of the 25-fs FLASH pulse, even when exposed to fluences that exceed the melt damage threshold by fifty times or more. We present a computational model for the FLASH laser-material interaction to analyze the extent to which the optics still function during the pulse. Comparison to experimental results obtained at FLASH shows good quantitative agreement.
\end{abstract}

Keywords: Damage threshold, x-ray optics, free-electron laser

\section{INTRODUCTION}

X-ray free-electron-lasers (XFELs) [1-4] will provide radiation of unprecedented peak brightness and full transverse coherence, enabling unique research possibilities for condensed matter physics, materials science, and structural biology. The currently operating soft-x-ray free electron laser FLASH [1] provides photon pulses of wavelength 13 to $48 \mathrm{~nm}$, and is expected to operate down to $6 \mathrm{~nm}$ soon. The hard-x-ray free electron lasers LCLS [2], SCSS [3], and Euro XFEL [4] will provide photon pulses of wavelengths down to $0.1 \mathrm{~nm}$. For all these FELs, the pulse length is $\leq 100 \mathrm{fs}$, and each pulse contains $10^{12}$ to $10^{13}$ photons, resulting in photon pulses of unprecedented peak brightness. The expected high output fluence and short pulse duration pose significant challenges to the optical components required to utilize XFEL beams, including radiation damage. Theoretical work on the design of robust optics has been discussed in the literature [5-9]. It is expected that high-melting-point, low-atomic-number materials will be most resistant to damage. It has been suggested that the fundamental mechanism that determines the damage threshold for single-pulse exposures in insulators is thermal melting [7,8]. For multiple-pulse exposures, the damage threshold is potentially lower due to fatigue effects associated with thermo-mechanical stresses [9], chemical changes, or phase transitions [10].

In recent experiments at FLASH it was demonstrated that the conventional damage threshold can be overcome by taking advantage of the extremely short pulse duration of the FELs [11]. It has been shown that during the pulse, only a limited amount of damage takes place, and the optic still functions. Only after the pulse is over is the optic destroyed. This is the same principle used for x-ray diffraction flash imaging of objects [12]. In this paper, we describe examples for "disposable" damage-resistant single-pulse optics used in soft x-ray FEL beamlines and their applications, especially focusing on multilayer mirrors. We will describe a model that is used to estimate the single-pulse damage resistance of optics. Finally, we describe how this principle can also be applied in the hard x-ray regime to other optical elements such as refractive lenses or x-ray zone plates.

\section{EXAMPLES FOR DAMAGE-RESISTANT SINGLE-PULSE OPTICS}

Several examples for damage-resistant single-pulse optics have been demonstrated recently at FLASH, including multilayer mirrors, spherical lenses, and apertures. In this Section we will review these findings. 


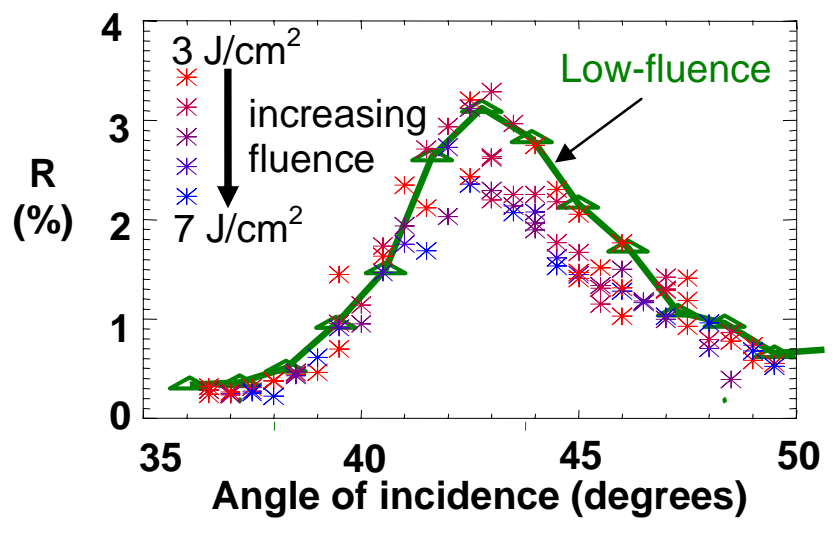

Fig. 1. Reflectivity as a function of the off-normal angle of incidence $\theta$ for different fluences. Data taken from Ref. [11].
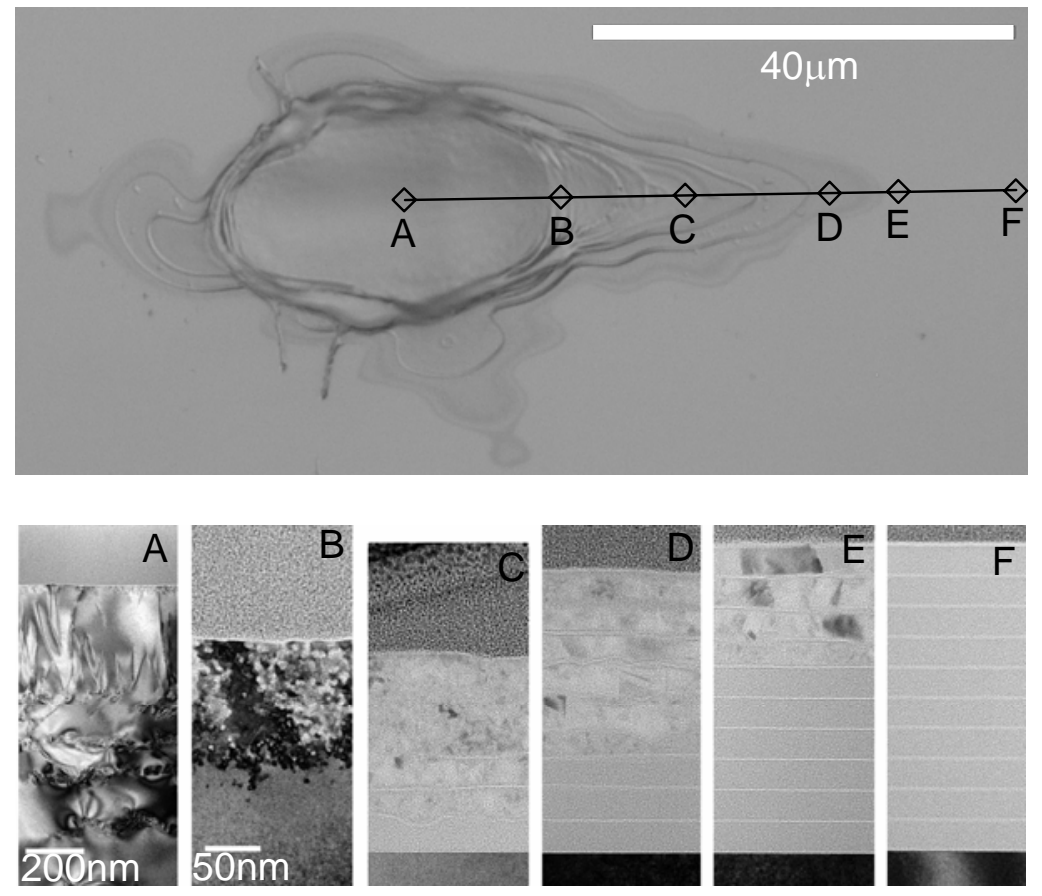

Fig. 2. (top) Nomarski differential interference contrast micrograph of the damaged multilayer surface. (bottom) TEM images of cross sections of the nanostructure at different positions within the FEL-exposed spot. The positions are as indicated in the top picture. The picture in the top and the bottom pictures A, C, and F were taken from Ref. [11].

\subsection{Multilayer mirrors}

The possibility of overcoming the damage barrier by applying short pulses has significant technological interest. For xrays, multilayer coatings can provide optics of higher numerical aperture (resulting in smaller focal spots) than uncoated mirrors. Extremely high focusing will be required for the application of XFELs to bio-molecular imaging or the creation and observation of extreme conditions in matter, such as exotic excited states of atoms and warm dense plasmas [13]. A common problem is the damage resistance of these multilayers when irradiated with multiple shots. If the survival of the multilayers for single shots is sufficient, significantly larger fluences can be tolerated. It was shown [11] that multilayer 
mirrors still functions during the pulse (Figure 1) even though they are completely destroyed after the pulse (Figure 2). In this example, the multilayer consisted of alternating $\mathrm{Si}$ and $\mathrm{C}$ layers and was irradiated with FLASH pulses at a wavelength of $32 \mathrm{~nm}$ at angles around 45 degrees.

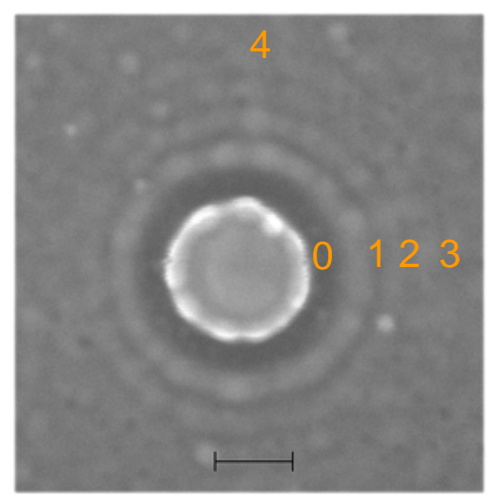

$100 \mathrm{~nm}$

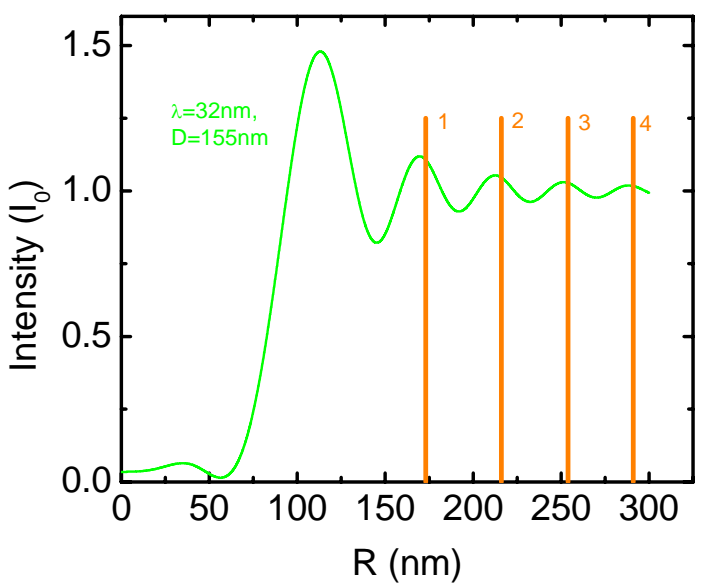

Fig. 3. (a) $\mathrm{SEM}$ image of a latex sphere on a $\mathrm{Si}_{3} \mathrm{~N}_{4}$ membrane after FLASH exposures. The dark rings correspond to depressions in the $\mathrm{Si}_{3} \mathrm{~N}_{4}$. (b) Calculated near-field diffraction pattern at the $\mathrm{Si}_{3} \mathrm{~N}_{4}$ surface.

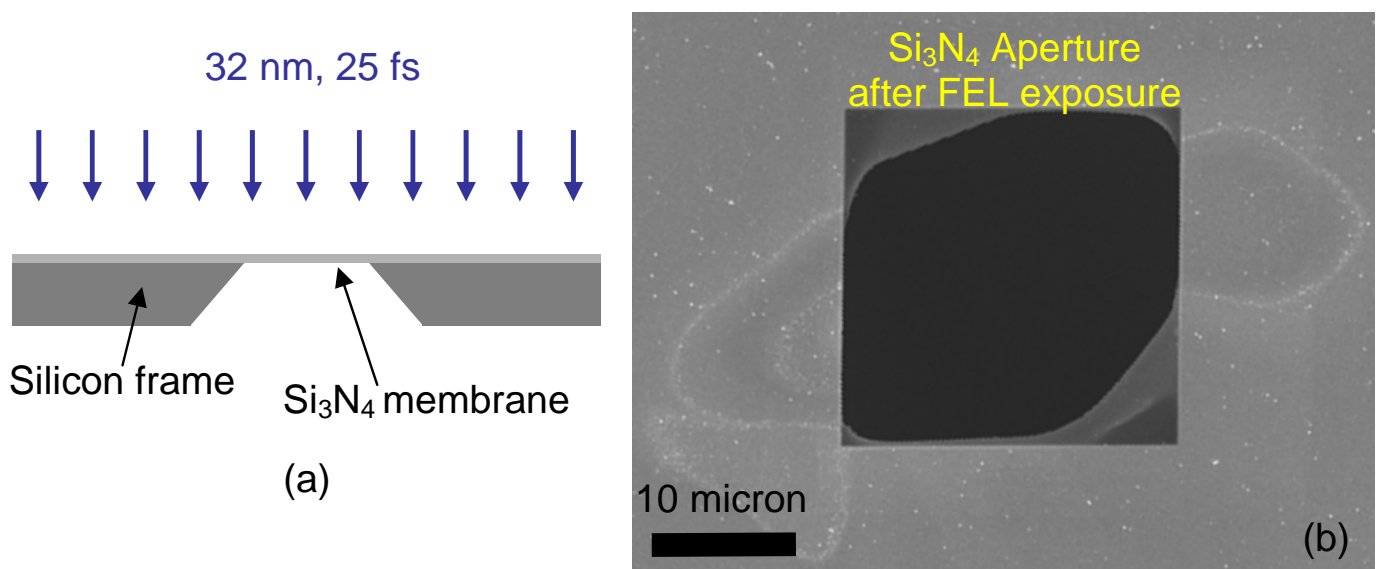

Fig. 4. Schematic of a Si/Si $\mathrm{N}_{4}$ aperture used in the FLASH imaging experiments. (b) SEM micrograph of a destroyed aperture after two FLASH exposures.

\subsection{Spherical nanolenses}

Polystyrene spheres act as diverging lenses at a wavelength of $32 \mathrm{~nm}$ since the index of refraction is less than one. We have demonstrated the functionality of such a lens at fluences that exceed the conventional damage threshold by depositing latex spheres on top of $20 \mathrm{~nm}$-thick $\mathrm{Si}_{3} \mathrm{~N}_{4}$ membranes and exposing them to the FLASH beam. We found that for a fluence range that causes mild damage to the $\mathrm{Si}_{3} \mathrm{~N}_{4}$ without destroying it, ring-shaped craters surrounding the spheres are formed, as shown in Figure 3 (a). The sphere is destroyed after the exposure to the $32 \mathrm{~nm}$ beam. As shown in Figure 3 (b), we can match these rings with maxima in the near-field diffraction pattern of the sphere. It is expected that these rings of high intensity also occurs at larger FLASH fluences, but the evidence is not available since the whole 
Si3N4 membrane is destroyed after the pulse. From these observations, we suggest that focusing concave-shaped nanolenses can be constructed and used with FELs for single high fluence pulses.

\subsection{Apertures}

In a number of diffractive imaging experiments at $\mathrm{FLASH}, \mathrm{Si}_{3} \mathrm{~N}_{4}$ windows that have been fabricated from silicon wafers covered with a thin layer of $\mathrm{Si}_{3} \mathrm{~N}_{4}$ using the anisotropic Si etchant $\mathrm{KOH}$, have been used to shape the beam. Figure 4 (a) shows a sketch of such an aperture. The gradual thickness variation of the silicon frame reduces the effect of the aperture on the diffraction pattern. We found that these apertures still function during the pulse but are destroyed afterwards. Figure 4 (b) shows such an aperture after exposure.

\section{LIMITATIONS OF SINGLE-PULSE OPTICS}

Even though single-pulse optics have a much larger damage tolerance than multiple-pulse optics, degradation of the optics during the pulse is still a concern for sufficiently large fluences or longer pulses. The FEL pulse heats the electrons, which can lead to changes in the optical properties of the materials. Even though the extent of large-scale atomic motion during the pulse is small, small-scale motion can potentially alter the crystal structure, and thereby the optical properties of the materials. In experiments at FLASH, indications were found that the optical properties do not change significantly during the pulse [14]. In the damage model that is described in the following Section we ignore changes in the index of refraction due to changes in the crystal structure factor and take only changes due to heating of the electrons into account.

\section{HYDRODYNAMIC MODEL FOR DAMAGE EXPERIMENTS AT FLASH}

We have gained a deeper understanding of the limitations of single-pulse optics through numerical simulations using hot dense plasma models. Since the samples enter the regime of warm-dense matter (WDM), which is generally not very well understood, we extended physical models originally developed for hot dense plasmas into the WDM regime. Since the relevant length scales in the optical elements are usually comparable to the wavelength, geometrical optics is generally not sufficient to describe the energy deposition, and wave optics needs to be considered. For example, for multilayer structures, the standing-wave intensity distribution was calculated using steady-state methods based on Fresnel equations [15]. We based our estimate of the complex index of refraction of the multilayer materials on published room-temperature solid-density opacities [16], corrected to higher temperatures and various densities by applying multiplicative correction factors based on an average-ion screened-hydrogen model [17]. The real part of the index was calculated from the opacities using the Kramers-Kronig dispersion formula. The soft x-ray beam is absorbed primarily by the electrons through bound-free absorption. Only when the electron temperature reaches $10 \mathrm{eV}$ does freefree absorption become larger than bound-free absorption in silicon. In carbon, this crossover occurs at an electron temperature of $35 \mathrm{eV}$ which was not reached in these experiments. Finally, we calculated the hydrodynamic explosion of the multilayer using the HYDRA radiation hydrodynamics code [18]. We use the QEOS equation-of-state (EOS) model to find the pressure and energy of the material [19]. For the conditions of our experiment $\left(\mathrm{T}_{\mathrm{e}} \leq 30 \mathrm{eV}, \mathrm{T}_{\mathrm{ion}}<<\mathrm{T}_{\mathrm{e}}\right.$, and solid density), the electrons dominate the EOS. They are described by a Thomas-Fermi model with an additional correction for chemical binding. Electron transport and electron-ion coupling are described in the relaxation time approximation, where the momentum transfer cross sections are calculated from Coulomb cross sections with cut-off parameters determined from partial-wave calculations. We found that heat conduction and radiation transport have little effect on the explosion dynamics during the pulse. 


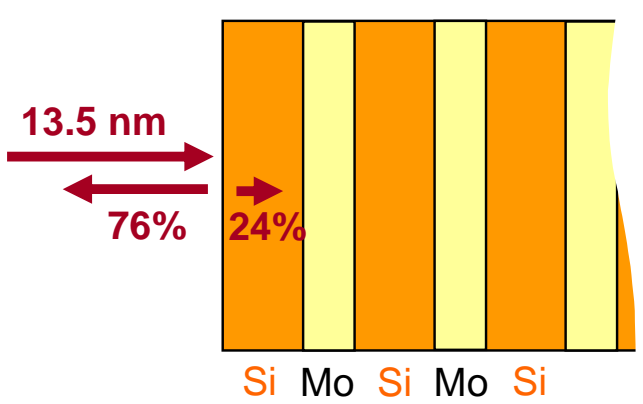

(a)
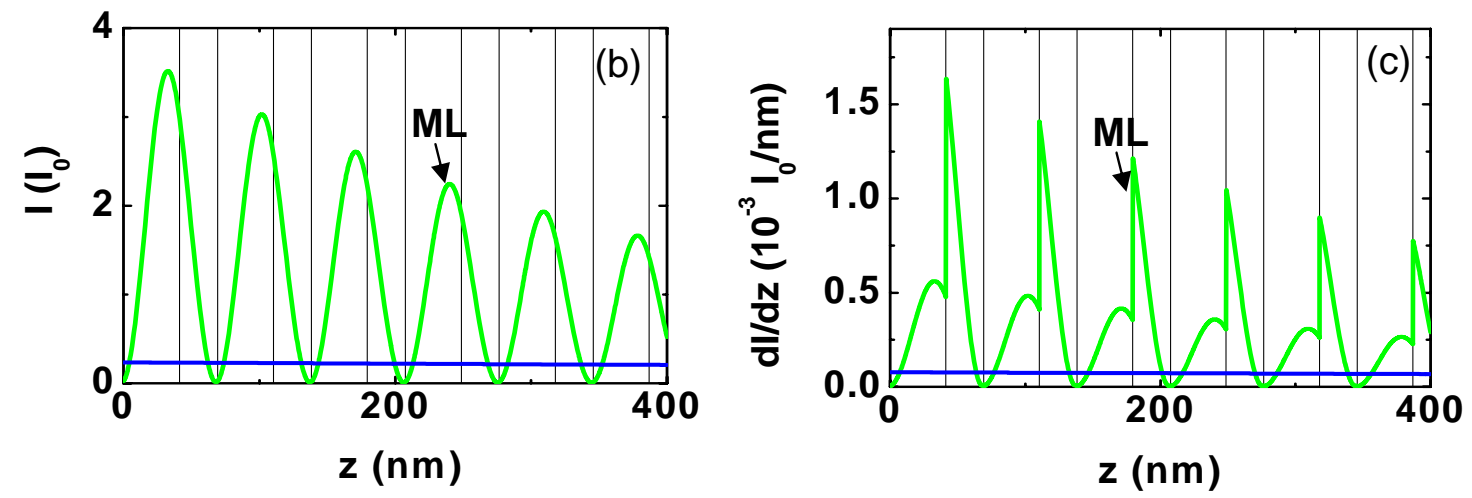

Fig. 5. (a) Schematic of a Mo/Si multilayer mirror. (b) Light intensity inside of the multilayer mirror. Overlaid is the intensity distribution inside of a homogeneous Mo/Si slab absorbing $24 \%$ of the beam. (c) Energy dose deposited in the multilayer mirrors. Overlaid is the dose inside of a homogeneous $\mathrm{Mo} / \mathrm{Si}$ slab.

\section{ANALYSIS OF DAMAGE-RESISTANT MULTILAYER MIRRORS}

We have used the hydrodynamic model to analyze the damage dynamics in resonant multilayer mirror structures used in some of the FLASH experiments. The deposition of the FLASH pulse energy in the mirror is governed by standingwave effects inside of the multilayer mirror. Figure 5 (a) shows a schematic of a Mo/Si multilayer irradiated at a wavelength of $13.5 \mathrm{~nm}$. For an optimized thickness ratio of the Mo and Si layers, the maximum theoretically achievable reflectivity is $76 \%$ for 50 bilayers. The remaining $24 \%$ of the incoming beam is absorbed in the multilayer mirror. Using an algorithm based on Fresnel equations [15], we calculated the beam intensity inside of the multilayer mirror as shown in Fig. 5 (b). Near resonance, the beam intensity is largest inside of the Si spacer layer, and smaller inside of the absorbing Mo layers. For comparison, in Fig. 5 (b) we also plotted the intensity distribution inside of a homogeneous $\mathrm{Mo} / \mathrm{Si}$ slab absorbing $24 \%$ of the beam, demonstrating the importance of wave effects in calculating the intensity distribution in structures with feature sizes that are comparable to the wavelength. Figure 5 (c) shows the energy dose deposited in the multilayer mirrors. Even though the intensity is lowest in the Mo layers, the dose is large there due to the high opacity of Mo. We also overlaid the equivalent dose inside of a homogeneous slab.

The fluence dependence of the reflectivity of a Si/C multilayer at $32.5 \mathrm{~nm}$ wavelength at an angle of incidence of 45 degrees was measured at FLASH [11]. The experimental results and our model predictions are reproduced in Fig. 6 . We found that the calculated reflectivity is somewhat higher than the measured value since the simulation results are shown for an angle of incidence of 45 degrees, whereas the experimental data is shown for angles between 44 and 47 degrees. The relative drop of the calculated reflectivity lies within the measured range. 


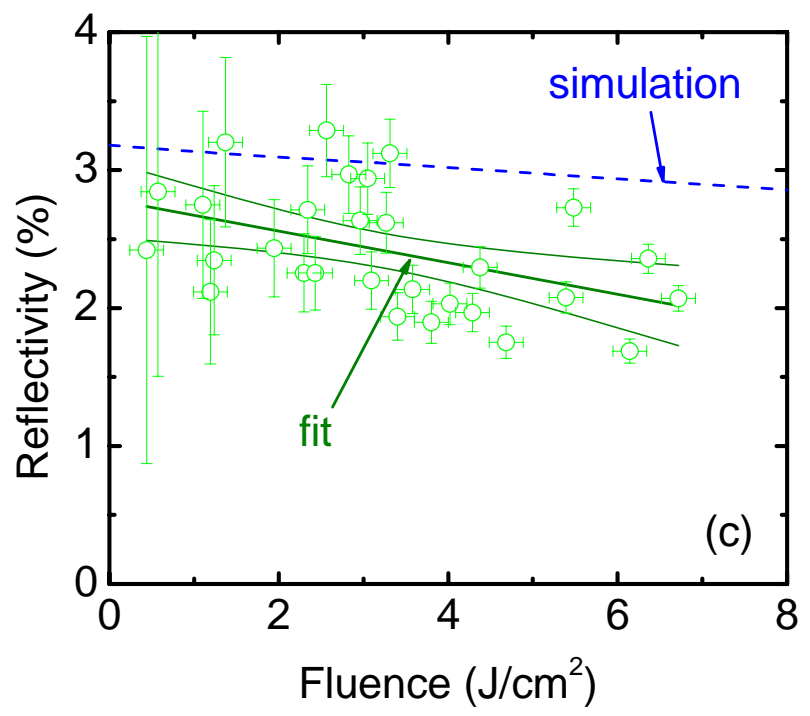

Fig. 6. Reflectivity of $\mathrm{Si} / \mathrm{C}$ multilayer calculated with hydrodynamic code and comparison to experiment. Figure taken from Ref. [11].

\section{DAMAGE-RESISTANT SINGLE-PULSE OPTICS FOR HARD X-RAYS}

So far we have described damage-resistant single-pulse optics in the soft x-ray regime. For hard x-rays, to date, the smallest x-ray focal spots of $30 \mathrm{~nm}$ diameter at $19.5 \mathrm{keV}$ photon energy have been achieved with thick multilayer structures, operating in a Laue (transmission) geometry [20]. It is predicted that these multilayer Laue lenses will produce spot sizes below $5 \mathrm{~nm}$, and hence could be used to focus XFEL pulses to achieve X-ray power densities of $3 \times 10^{22} \mathrm{~W} / \mathrm{cm}^{2}$, assuming anticipated XFEL light output [2]. This corresponds to electric-field strengths close to the Schwinger critical field, at which spontaneous electron-positron pair creation is expected to take place [21]. These lenses are only several hundred micrometers in diameter, and so will necessarily be exposed to high incident power densities, where damage will occur, but not before carrying out their function.

Beam splitters based on Bragg reflection from single crystals hold the promise to facilitate single-color pump-probe experiments in the hard x-ray regime. For certain angles of incidence, hard x-rays are specularly reflected (and refracted) from single crystals. It is anticipated that significantly larger fluences than the melt fluences can be tolerated if the application of single-pulse optics is acceptable.

\section{SUMMARY AND CONCLUSIONS}

We have demonstrated that many optical elements still function during ultrashort pulses, even through they are completely destroyed afterwards. Examples of such damage-resistant single-pulse optics that have been demonstrated at the FLASH facility include multilayer mirrors, beam apertures, and refractive lenses. We have developed a model to predict the limitations of these single-pulse optics. In the experiments at FLASH, the samples were heated into the warm-dense-matter regime. In this regime, the index of refraction is very difficult to calculate and the EOS is not very well known. For the calculation of the energy deposition, diffraction effects have to be taken into account for certain geometries, for example for multilayer mirrors or spheres on membranes that are comparable in size to the wavelength. We found that our model agrees with experimental results collected at FLASH, and that it can be used to predict the performance of disposable, damage-resistant single pulse optics. 


\section{ACKNOWLEDGEMENTS}

This work was performed under the auspices of the U.S. Department of Energy by the University of California, Lawrence Livermore National Laboratory under contract No. W-7405-Eng-48.

\section{REFERENCES}

1. V. Ayvazyan, et al., Eur. Phys. J. D 37, 297 (2006).

2. Linac Coherent Light Source (LCLS) Design Study Report, SLAC-R-521, 1998, National Technical Information Services, 5285 Port Royal Road, Springfield, Virginia, 22161.

3. T. Shintake et. al., Nucl. Inst. and Meth. A507, 382 (2003); http://www-xfel.spring8.or.jp.

4. "XFEL Technical Design Report", M. Altarelli et al. (eds.), DESY 2006-067, DESY, Hamburg, August 2006.

5. R. Tatchyn, J. Arthur, R. Boyce, T. Cremer, A. Fasso, J. Montgomery, V. Vylet, D. Walz, R. Yotam, A.K. Freund, and M.R. Howells, Proc. SPIE 3154, 174 (1998).

6. R. Bionta, LCLS Technical Note LCLS-TN-00-3, available at http://www-ssrl.slac.stanford.edu/lcls/technotes/.

7. R.A. London, R.M. Bionta, R.O. Tatchyn, and S. Roesler, Proc. SPIE 4500, 51 (2001).

8. A. Wootton, J. Arthur, T. Barbee, R. Bionta, R. London, H.-S. Park, D. Ryutov, E. Spiller, and R. Tatchyn, Proc. SPIE 4500, 113 (2001).

9. D.D. Ryutov, Rev. Sci. Instr. 74, 3721 (2003).

10. L. Juha, M. Bittner, M. De Grazia, J. Feldhaus, J. Gaudin, S. Guizard, S. Jacobi, M. Kozlová, J. Krása, J. Krzywinski, H. Merdji, C. Michaelsen, T. Mocek , R. Nietubyc, M. Jurek, J. Polan, A. R. Präg, B. Rus, R. Sobierajski, B. Steeg-Keitel, M. Störmer, M. Stupka, V. Vorlíček, J. Wiesmann, J. Wild, Proc. SPIE 5917,91 (2005).

11. S. Hau-Riege, H. Chapman, J. Krzywinski, R. Sobierajski, R. London, R. Bionta, M. Bergh, C. Caleman, R. Nietubyc, L. Juha et al., Phys. Rev. Lett. 98, 145502 (2007).

12. H. Chapman, A. Barty, M. Bogan, S. Boutet, M. Frank, S. Hau-Riege, S. Marchesini, B. Woods, S. Bajt, W. Brenner et al., Nature Physics 2, 839 (2006).

13. G.K. Shenoy and J. Stöhr, eds., "LCLS - The First Experiments", SSRL (2000), available at the web site http://www-ssrl.slac.stanford.edu/lcls/papers/lcls_experiments_2.pdf.

14. J. Krzywinski et al. (unpublished).

15. D.L. Windt, Comp. Phys. 12, 360 (1998).

16. B.L. Henke, E.M. Gullikson, and J.C. Davis, Atom. Nucl. Data Tabl. 54, 181 (1993).

17. R.M. More, J. Quant. Spectrosc. Radiat. Transf. 27, 345 (1982).

18. M.M. Marinak, Phys. Plasmas. 8, 2275 (2001).

19. R.M. More et al., Phys. Fluids 31, 3059 (1988).

20. H.C. Kang et al., Phys. Rev. Lett. 96, 127401 (2006).

21. M. Marklund and P.K. Shykla, Rev. Mod. Phys. 78, 591 (2006). 